\title{
Concurrent fine needle aspirations and core needle biopsies: a comparative study of substrates for next-generation sequencing in solid organ malignancies
}

Sinchita Roy-Chowdhuri ${ }^{1}$, Hui Chen ${ }^{1}$, Rajesh R Singh ${ }^{2}$, Savitri Krishnamurthy ${ }^{1}$, Keyur P Patel ${ }^{2}$, Mark J Routbort ${ }^{2}$, Jawad Manekia ${ }^{2}$, Bedia A Barkoh ${ }^{2}$, Hui Yao ${ }^{3}$, Sharjeel Sabir ${ }^{4}$, Russell R Broaddus ${ }^{1}$, L Jeffrey Medeiros ${ }^{2}$, Gregg Staerkel ${ }^{1}$, John Stewart ${ }^{1}$ and Rajyalakshmi Luthra ${ }^{2}$

${ }^{1}$ Department of Pathology, Division of Pathology and Laboratory Medicine, The University of Texas MD Anderson Cancer Center, Houston, TX, USA; ${ }^{2}$ Department of Hematopathology, Division of Pathology and Laboratory Medicine, The University of Texas MD Anderson Cancer Center, Houston, TX, USA; ${ }^{3}$ Department of Bioinformatics and Computational Biology, The University of Texas MD Anderson Cancer Center, Houston, TX, USA and ${ }^{4}$ Department of Interventional Radiology, Division of Diagnostic Imaging, The University of Texas MD Anderson Cancer Center, Houston, TX, USA

Minimally invasive procedures, such as fine needle aspiration and core needle biopsy, are commonly used for the diagnosis in solid organ malignancies. In the era of targeted therapy, it is crucial for molecular testing to be performed on these limited volume specimens. Although several recent studies have demonstrated the utility of small biopsy specimens for molecular testing, there remains debate as to whether core needle biopsy specimens are more reliable than fine needle aspiration for molecular studies. In this study, we reviewed concurrently acquired fine needle aspiration and core needle biopsy samples $(n=24)$, and compared overall cellularity, tumor fraction, and the results of next-generation sequencing. All somatic mutations detected in core needle biopsy samples were also detected in fine needle aspiration samples. The estimated tumor fraction was significantly higher in fine needle aspiration smears than core needle biopsy samples $(P=0.003)$, whereas the overall DNA yield from smears was significantly lower than that obtained from the core needle biopsy specimens $(P=0.01)$. The normalized average amplicon coverage for the genes analyzed was significantly higher in cytology smears than paired core needle biopsy samples, with lower numbers of failed amplicons and higher overall mutation allelic frequencies seen in the former. We further evaluated $\mathbf{1 0 0}$ malignant fine needle aspiration and core needle biopsy samples, acquired concurrently, for overall cellularity and tumor fraction. Overall cellularity and tumor fraction of fine needle aspiration samples was significantly higher than concurrently acquired core needle biopsy samples $(P<0.001)$. In conclusion, we show that fine needle aspiration samples frequently provide better cellularity, higher tumor fraction, and superior sequencing metrics than concurrently acquired core needle biopsy samples. Cytologic specimens, therefore, should be better integrated into routine molecular diagnostics workflow to maximize limited tissues for clinically relevant genomic testing.

Modern Pathology (2017) 30, 499-508; doi:10.1038/modpathol.2016.228; published online 13 January 2017

Correspondence: Dr S Roy-Chowdhuri, MD, PhD, Department of Pathology, Division of Pathology and Laboratory Medicine, The University of Texas MD Anderson Cancer Center, 1515 Holcombe Blvd. Unit 85, Houston, TX 77030, USA.

E-mail: sroy2@mdanderson.org

Received 18 August 2016; revised 14 November 2016; accepted 18 November 2016; published online 13 January 2017
Ancillary molecular testing is being used increasingly to direct personalized cancer therapy for solid organ malignancies. In a large fraction of patients, these diagnoses are established by using small tissue biopsy samples obtained through minimally invasive procedures, including fine needle aspiration and core needle biopsy. Therefore, it is critical to be able 
to perform molecular studies on these small samples to screen for predictive and prognostic markers and provide therapeutic targets.

Currently, both fine needle aspiration and core needle biopsy are used for obtaining diagnostic material and the choice of sampling method is largely dictated by the size and location of the lesion, the institutional practice, and the skill and preference of the operator. ${ }^{1}$ Fine needle aspiration samples are generally obtained using 20-25 gauge needles, with or without rapid on-site evaluation for adequacy, and are often preferred for sampling small and/or deep seated lesions, tumors adjacent to at-risk structures such as blood vessels, and in situations where the needle is passed through the bronchial or bowel wall. ${ }^{2,3}$ Core needle biopsy samples are acquired using larger 14-20 gauge needles and provide a tissue core with preservation of tissue architecture that can be used for ancillary studies. ${ }^{3}$ Both sampling methods provide distinct advantages and limitations that are frequently complementary. Therefore, in a large number of practices, including our own institution, both methods are utilized concurrently as a diagnostic modality in amenable lesions.

Although several studies have demonstrated the utility of cytology specimens for molecular analysis, there has been some controversy regarding the adequacy of fine needle aspiration specimens $s^{4,5}$ and in most institutions a core needle biopsy specimen is preferred over an fine needle aspiration sample for molecular testing. ${ }^{2,6} \mathrm{~A}$ few studies have addressed the adequacy of cytology fine needle aspiration samples in comparison with surgical biopsy/resection samples; ${ }^{7-14}$ however, relatively few studies have systematically evaluated concurrently acquired fine needle aspiration and core needle biopsy samples for cellularity, tumor fraction, and downstream molecular analysis.

In this study, we reviewed 24 concurrently acquired fine needle aspiration and core needle biopsy samples to compare cellular adequacy, tumor fraction, DNA yield, and the results of nextgeneration sequencing mutational analysis. In addition, we reviewed 100 consecutive concurrently acquired malignant fine needle aspiration and core needle biopsy samples for overall cellularity and tumor fraction.

\section{Materials and methods}

\section{Tumor Sample Acquisition and Processing}

The institutional pathology database was searched for all patients undergoing in-house concurrent image-guided percutaneous fine needle aspiration and core needle biopsy sampling, for which adequate diagnostic material was obtained for both fine needle aspiration and core needle biopsy specimens. We identified 24 consecutive malignant cases
(December 2014 to January 2015) with concurrent fine needle aspiration and core needle biopsy samples that had next-generation sequencing results available on either one of the samples as part of the routine clinical work-up of the patient and with tissue available in the concurrent sample to perform matched next-generation sequencing. Two cases were rejected following initial review owing to inadequate cellularity in the fine needle aspiration sample. The selected samples included adenocarcinoma $(n=17)$, carcinoma not otherwise specified $(n=4)$, squamous cell carcinoma $(n=2)$, and melanoma $(n=1)$ from a variety of sites including lymph node $(n=7)$, liver $(n=7)$, lung $(n=6)$, soft tissue $(n=2)$, bone $(n=1)$, and adrenal gland $(n=1)$. An additional 100 consecutive malignant cases (June-July 2013) with concurrent in-house imageguided fine needle aspiration and core needle biopsy samples were also selected for morphologic evaluation. The specific diagnoses of these cases included: adenocarcinoma $(n=51)$, squamous cell carcinoma $(n=18)$, poorly differentiated carcinoma $(n=11)$, melanoma $(n=7)$, neuroendocrine carcinoma $(n=5)$, small cell carcinoma $(n=3)$, sarcoma $(n=3)$, chordoma $(n=1)$, and germ cell tumor $(n=1)$. Sites of the lesions included lung $(n=34)$, liver $(n=23)$, soft tissue $(n=19)$, lymph node $(n=17)$, bone $(n=4)$, adrenal gland $(n=2)$, and kidney $(n=1)$. The study was approved by the Institutional Review Board at our institution. Sampling was performed by interventional radiologists using ultrasound or computed tomography guidance with coaxial technique. In total, 17-19 gauge guide needles were placed adjacent to the targeted lesion and used to introduce 20-25 gauge needles for fine needle aspiration sampling (2-3 passes) and/or 16-20 gauge needles for obtaining core needle biopsy samples (3-4 cores) through the guide needle. In general, fine needle aspiration was performed first, followed by core needle biopsy sampling. However, core needle biopsy was often performed first, in subcentimeter tumors at risk of being obscured by hemorrhage from fine needle aspiration.

Direct smear preparations were made from fine needle aspirated tissue and stained using the DiffQuik and Papanicolaou methodology (previously described $^{15}$ ) for immediate assessment by a cytopathologist. After preparing smears, aspiration needles were rinsed in RPMI and cytospin or cell block preparations (ethanol/formalin-fixed and paraffinembedded $)^{6}$ were prepared for further evaluation. Fine needle aspiration samples evaluated for this study included direct smears for all cases with cell block preparations in 53 cases. The core needle biopsy samples were fixed in $10 \%$ neutral buffered formalin, paraffin-embedded, sectioned at a thickness of $4 \mu \mathrm{m}$, and stained with hematoxylin and eosin (H\&E). 


\section{Assessment of Sample Cellularity and Tumor Fraction}

The samples were visually evaluated by light microscopy and scored as described below. As most next-generation sequencing assays require approximately between 1000 cells (eg, Ion Torrent PGM) and 5000 cells (eg, Illumina MiSeq), ${ }^{16}$ the overall cellularity for smears scored based on the entire slide was: high, at least 1 or 2 slides with $>5000$ cells; moderate, 1000-5000 cells; low, $<1000$ cells. Overall cellularity assessments for cell blocks were not performed. Tumor fraction assessments were based upon selected tumor-rich areas within the slide. Smears and cell blocks were scored separately for tumor fraction (percentage of tumor cells relative to all cells in selected tumor-enriched areas) and when both preparations were available for review, a combined tumor fraction was generated for each case. All fine needle aspiration samples were evaluated independently by two cytopathologists (SR-C and JS). Tumor fraction assessments that were within $10 \%$ difference were averaged; any case that had $>10 \%$ discordance were reviewed together by the two cytopathologists to arrive at a consensus opinion.

Core needle biopsies were scored for overall cellularity and tumor fraction based on two H\&Estained section levels (levels 1 and 8) independently by two pathologists (SR-C and HC). Cellularity for core needle biopsy samples was scored for the entire slide as: high $>2000$ per H\&E slide; moderate 300-2000 per H\&E slide; low $<300$ per H\&E slide. The cellularity assessment criteria for core needle biopsy samples was different from the fine needle aspiration samples based on our previous experience, as (a) core needle biopsy FFPE sections are $4 \mu \mathrm{m}$ thick and do not contain the entire nuclei resulting in lower DNA yield than the whole cells retrieved from direct smears; and (b) sectioning through the paraffin block results in loss of cellular material in subsequent sections contributing to lower DNA yield than what is estimated on a representative H\&E section. Tumor fraction assessments were based upon selected tumor-rich areas within the slide. Similar to fine needle aspiration samples, any case that had discordance was reviewed again by both pathologists to reach a consensus opinion. Tumor fraction assessments within $10 \%$ difference were averaged and any case that had $>10 \%$ discordance between the two pathologists were adjudicated by a re-review by both pathologists.

\section{Mutation Testing and Data Analysis}

The 24 paired fine needle aspiration and core needle biopsy samples were analyzed by next-generation sequencing using the Ion Torrent PGM (Thermo Fisher Scientific, MA) as previously described.6,17 Fine needle aspiration smears that were selected for sequencing were photographed (representative fields) prior to extraction for digital archiving. In addition, all cases had additional smears and/or cell block material with diagnostic material retained on file for archival purposes. DNA was extracted from circled tumor-rich areas of direct smears (Diff-Quik and Papanicolaou stained) and formalin-fixed paraffin-embedded unstained tissue sections prepared from core needle biopsy paraffin blocks ${ }^{17}$ using PicoPure (Arcturus, Mountain View, CA) and quantified using a Qubit DNA high sensitivity assay kit (Thermo Fisher Scientific) as per manufacturer's guidelines. An amplicon library was prepared using DNA from each sample using the IT Ampliseq Cancer Panel primers and quantified using the Bioanalyzer High Sensitivity DNA Chip (Agilent Technologies, Santa Clara, CA). The manufacturerrecommended input DNA for the next-generation sequencing assay is $10 \mathrm{ng}$ (DNA concentration of $0.0009 \mu \mathrm{g} / \mathrm{\mu l}$ ). If DNA yield was high, appropriate dilutions were made; however, if DNA yield was suboptimal, DNA was used as-is without any further dilution. Emulsion PCR was performed manually using the Ion Xpress Template Kit (Thermo Fisher Scientific) followed by manual breaking of the emulsion to isolate the ion spheres (ISPs). The quality of the DNA following PCR was measured using the Qubit IonSphere Quality control kit (Thermo Fisher Scientific). Selective ISPs with DNA were isolated and sequenced on an Ion 318 Chip. A minimum of 300000 reads with a quality score of AQ20 (1 misaligned base per 100 bases) was used as a measure of successful sequencing with a minimum coverage of $\times 250$ for a wild-type call. ${ }^{6}$

Sequence alignment and base calling was performed by Torrent Suite software v3.6.2 (Thermo Fisher Scientific) using Human Genome Build 19 (Hg19) as the reference sequence. IT Variant Caller Plugin software v3.5.59049 (Thermo Fisher Scientific) was used for the detection of variants and the variants were visualized using the Integrative Genomics Viewer (IGV). An in-house custom software (OncoSeek) was used to interface the data generated by Variant Caller with IGV to visualize the alignment and mutation detected in IGV, and annotate the sequencing results. As paired normal tissue samples were not available for analysis in all patients, variants were annotated as somatic mutations or possible germline variants based on a literature survey (including somatic mutation information as reported in COSMIC (http://cancer. sanger.ac.uk/cancergenome/projects/cosmic/) and The Single Nucleotide Polymorphism Database (dbSNP) (http://www.ncbi.nlm.nih.gov/SNP/). Any mutant allele frequency of $<10 \%$ was reviewed in conjunction with the paired sample as well as in context of the tumor type and estimated tumor fraction in the sample. 


\section{Statistical Analysis}

The Wilcoxon rank-sum test and McNemar's $\chi^{2}$-test were used to test against the null hypothesis of no difference between the paired samples for continuous variable and categorical variable, respectively. The test results were considered to be statistically significant at a $P$-value $<0.05$.

\section{Results}

We reviewed 24 concurrently acquired fine needle aspiration and core needle biopsy samples that had next-generation sequencing mutational analysis performed. We compared DNA yield, tumor fraction, and the results of next-generation sequencing in these samples, including a variety of sequencing metrics, such as variant calls, mutation allelic frequencies, average amplicon coverage, and number of failed amplicons.

The estimated tumor fraction was significantly higher in fine needle aspiration smears than core needle biopsy samples $(P=0.003)$; however, the overall DNA yield from smears was significantly lower than that obtained from core needle biopsy specimens $(P=0.01)$ (Table 1$)$. All somatic mutations detected in core needle biopsy samples were detected in concurrent fine needle aspiration samples (Table 2). Details of specific mutations can be found in Supplementary Table 1. The overall mutation allelic frequencies observed in fine needle aspiration samples were higher than those in the concurrent core needle biopsy samples (Figure 1 and Table 2). To evaluate the quality of sequencing for the paired samples, we compared the average amplicon coverage by normalizing to the total number of reads in each case. The normalized average amplicon coverage for all 50 genes analyzed, as well as that of selected genes of relevance (BRAF, KRAS, NRAS, HRAS, EGFR, PIK3CA, KIT, $P T E N$, and TP53) was significantly higher in fine needle aspiration samples than in paired core needle biopsy samples $(P=0.025$ and 0.014 ,

Table 1 Tumor fraction and DNA yield from concurrently acquired fine needle aspiration and core needle biopsy samples

\begin{tabular}{lccc}
\hline & $\begin{array}{c}\text { FNA smears } \\
(\mathrm{n}=24)\end{array}$ & $\begin{array}{c}\text { CNB } \\
(\mathrm{n}=24)\end{array}$ & P-value \\
\hline $\begin{array}{l}\text { Tumor fraction } \\
\text { Mean }\end{array}$ & $54 \%$ & $39 \%$ & \\
Median & $60 \%$ & $30 \%$ & $P=0.003$ \\
Range & $25-90 \%$ & $20-70 \%$ & \\
DNA yield & & & \\
Mean & $6.6 \mathrm{ng} / \mu \mathrm{l}$ & $17.5 \mathrm{ng} / \mu \mathrm{l}$ & \\
Median & $3.6 \mathrm{ng} / \mu \mathrm{l}$ & $12.9 \mathrm{ng} / \mu \mathrm{l}$ & $P=0.01$ \\
Range & $0.36-21 \mathrm{ng} / \mu \mathrm{l}$ & $0.27-55 \mathrm{ng} / \mu \mathrm{l}$ & \\
\hline
\end{tabular}

Abbreviations: CNB, core needle biopsy; FNA, fine needle aspiration. respectively) (Table 3). In addition, the cytology samples had lower numbers of under-performing amplicons than the core needle biopsy samples, with an average of 0.46 failed amplicons in fine needle aspiration samples vs 2.25 in core needle biopsy samples (Table 3).

To further evaluate our findings, we selected an additional 100 paired samples of consecutive imageguided procedures from solid organ malignancies for morphologic review and visual estimation of overall cellularity and tumor fraction. The overall cellularity of the 100 cases reviewed was as follows: fine needle aspiration smears, high: $60 \%$; moderate: $33 \%$; low: $7 \%$, vs core needle biopsy samples high: $15 \%$; moderate: 58\%; low: 27\% (Figure 2). The overall cellularity of fine needle aspiration samples was significantly higher than that of the concurrent core needle biopsy samples $(P<0.001)$ with cases of adenocarcinoma, squamous cell carcinoma, poorly differentiated carcinoma, and neuroendocrine carcinoma/small cell carcinoma typically showing higher cellularity on the cytology smears than the concurrent core needle biopsy samples (49 vs $4 \% ; 78$ vs $33 \%$; 82 VS $0 \%$; and 100 VS 25\%, respectively) (Table 4). Metastatic tumors to the liver or lymph nodes typically showed higher cellularity on fine needle aspiration than core needle biopsy samples (Table 5).

We also compared the tumor cellularity between the fine needle aspiration and core needle biopsy samples by estimating tumor fraction based on tumor-enriched (circled) areas on the slides. Fine needle aspiration samples showed higher tumor fraction than concurrent core needle biopsy samples $(P<0.001)$, with $83 \%$ of fine needle aspiration samples having $40 \%$ or higher tumor fraction vs $54 \%$ of core needle biopsy samples (Figure 3 and Table 6). A significantly higher number of core needle biopsy samples had a tumor fraction $<20 \%$, which falls below our threshold for next-generation sequencing molecular testing $(P<0.001)$ (Table 6). Half of these low tumor fraction core needle biopsy samples $(n=7)$ had $10 \%$ or less-tumor cellularity. In the subset of 14 core needle biopsy samples with a $<20 \%$ tumor fraction, the concurrent fine needle aspiration had five samples with high cellularity and a median tumor fraction of $60 \%$ (range $40-80 \%$ ), seven samples with moderate cellularity and median tumor fraction of $60 \%$ (range 25-90\%), and two samples with low cellularity and median tumor fraction of $38 \%$ (range $35-40 \%$ ). There were also 14 core needle biopsy samples with a borderline adequate tumor fraction ranging between 20 and $25 \%$. Of these, eight had concurrent fine needle aspiration samples with high cellularity and a median tumor fraction of $75 \%$ (range 55-90\%) and six showed moderate cellularity and median tumor fraction of $43 \%$ (range $30-85 \%)$. 
Table 2 Mutational analysis characteristics of paired fine needle aspiration and core needle biopsy samples

\begin{tabular}{|c|c|c|c|c|c|c|c|c|c|c|c|c|}
\hline$P t$ & Site & $D x$ & Sample & Tumor \% & No. of slides used & $D N A$ yield $(n g / \mu l)$ & Input DNA (ng) & $M A F \# 1$ & $M A F \# 2$ & $M A F \# 3$ & $M A F \# 4$ & $M A F \# 5$ \\
\hline \multirow[t]{2}{*}{1} & LN & CRC & FNA & $80 \%$ & 2 smears & 14.1 & 13.6 & $A P C, 65 \%$ & & & & \\
\hline & & & CNB & $30 \%$ & 12 uss & 13.5 & 13.6 & $A P C, 35 \%$ & & & & \\
\hline \multirow[t]{2}{*}{2} & $\mathrm{LN}$ & SqCC & FNA & $90 \%$ & 1 smear & 2.9 & 13.6 & PIK $3 C A, 32 \%$ & & & & \\
\hline & & & $\mathrm{CNB}^{\mathrm{a}}$ & $60 \%$ & 12 uss & 0.6 & 7.7 & PIK $3 C A, 27 \%$ & & & & \\
\hline \multirow[t]{2}{*}{3} & $\mathrm{LN}$ & Lung ADC & FNA & $60 \%$ & 4 smears & 2.8 & 13.6 & $E G F R, 49 \%$ & PDGFRA,39\% & & & \\
\hline & & & CNB & $30 \%$ & 10 uss & 13.5 & 13.6 & $E G F R, 21 \%$ & PDGFRA,45\% & & & \\
\hline \multirow[t]{2}{*}{4} & Lung & $\mathrm{ADC}$ & FNA & $50 \%$ & 4 smears & 2.7 & 13.6 & KRAS,48\% & & & & \\
\hline & & & $\mathrm{CNB}$ & $20 \%$ & 8 uss & 4 & 13.6 & KRAS, $12 \%$ & & & & \\
\hline \multirow[t]{2}{*}{5} & Liver & $\mathrm{Br} \mathrm{ADC}$ & FNA & $70 \%$ & 2 smears & 6.3 & 13.6 & PIK3CA,5\% & TP53,9\% & $C D K N 2 A, 16 \%$ & & \\
\hline & & & CNB & $60 \%$ & 10 uss & 1.3 & 13.6 & PIK $3 C A, 3 \%$ & TP53,4\% & CDKN2A, $1 \%$ & & \\
\hline \multirow[t]{2}{*}{6} & Liver & $\mathrm{MM}$ & FNA $^{\mathrm{a}}$ & $60 \%$ & 3 smears & 0.6 & 6.6 & GNA11,22\% & & & & \\
\hline & & & CNB & $70 \%$ & 6 uss & 1.2 & 13.6 & GNA11,24\% & & & & \\
\hline \multirow[t]{2}{*}{7} & $\mathrm{LN}$ & CRC & FNA & $70 \%$ & 1 smear & 12.8 & 13.6 & TP53,79\% & & & & \\
\hline & & & CNB & $30 \%$ & 12 uss & 7.2 & 13.6 & TP53,19\% & & & & \\
\hline \multirow[t]{2}{*}{8} & Lung & ADC & FNA & $60 \%$ & 5 smears & 1.7 & 13.6 & KRAS, $24 \%$ & PIK $3 C A, 20 \%$ & $C D K N 2 A, 16 \%$ & TP53,7\% & ТР53,6\% \\
\hline & & & $\mathrm{CNB}$ & $50 \%$ & 7 uss & 25.7 & 27.3 & KRAS,20\% & PIK $3 C A, 18 \%$ & $C D K N 2 A, 31 \%$ & TP53,21\% & TР53,1\% \\
\hline \multirow[t]{2}{*}{9} & Liver & CRC & FNA & $70 \%$ & 3 smears & 14.3 & 13.6 & $K R A S, 38 \%$ & $A P C, 37 \%$ & TP53,55\% & & \\
\hline & & & $\mathrm{CNB}$ & $60 \%$ & 15 uss & 42.6 & 40.9 & $K R A S, 25 \%$ & $A P C, 25 \%$ & TP53,33\% & & \\
\hline \multirow[t]{2}{*}{10} & Lung & ADC & FNA & $70 \%$ & 4 smears & 5.7 & 13.6 & TP53,82\% & & & & \\
\hline & & & $\mathrm{CNB}$ & $30 \%$ & 10 uss & 3.9 & 13.6 & TP53,54\% & & & & \\
\hline \multirow[t]{2}{*}{11} & Lung & $\mathrm{ADC}$ & FNA & $50 \%$ & 2 smears & 7 & 13.6 & $E G F R, 24 \%$ & $E G F R, 27 \%$ & & & \\
\hline & & & CNB & $50 \%$ & 10 uss & 2.3 & 13.6 & $E G F R, 12 \%$ & $E G F R, 15 \%$ & & & \\
\hline \multirow[t]{2}{*}{12} & Liver & CRC & FNA & $40 \%$ & 3 smears & 4.4 & 13.6 & PIK $3 C A, 72 \%$ & $K R A S, 32 \%$ & $A P C, 53 \%$ & & \\
\hline & & & $\mathrm{CNB}^{\mathrm{a}}$ & $30 \%$ & 12 uss & 0.3 & 3.2 & PIK $3 C A, 48 \%$ & KRAS, $22 \%$ & $A P C, 25 \%$ & & \\
\hline \multirow{2}{*}{13} & Adr & Lung ADC & FNA & $50 \%$ & 3 smears & 15.6 & 27.3 & KRAS,71\% & TP53,70\% & & & \\
\hline & & & CNB & $50 \%$ & 10 uss & 25.6 & 27.3 & $K R A S, 31 \%$ & TP53,24\% & & & \\
\hline \multirow[t]{2}{*}{14} & Liver & $\mathrm{Br} A D C$ & FNA & $70 \%$ & 3 smears & 21 & 27.3 & PIK $3 C A, 49 \%$ & & & & \\
\hline & & & CNB & $30 \%$ & 10 uss & 24.6 & 27.3 & PIK $3 C A, 60 \%$ & & & & \\
\hline \multirow{2}{*}{15} & $\mathrm{LN}$ & Lung ADC & FNA & $30 \%$ & 5 smears & 18.2 & 27.3 & ERBB2,14\% & TP53,8\% & & & \\
\hline & & & CNB & $40 \%$ & 15 uss & 55 & 54.5 & $E R B B 2,24 \%$ & TP53,19\% & & & \\
\hline \multirow[t]{2}{*}{16} & Bone & RCC & FNA & $30 \%$ & 3 smears & 1.2 & 13.6 & VHL, $19 \%$ & & & & \\
\hline & & & CNB & $30 \%$ & 8 uss & 10 & 13.6 & VHL,15\% & & & & \\
\hline \multirow{2}{*}{17} & ST & UroCA & FNA $^{\mathrm{a}}$ & $30 \%$ & 2 smears & 0.5 & 6.3 & TP53,73\% & & & & \\
\hline & & & CNB & $22 \%$ & 15 uss & 9.6 & 13.6 & TP53,25\% & & & & \\
\hline \multirow[t]{2}{*}{18} & Lung & PDC & FNA & $30 \%$ & 4 smears & 2.3 & 13.6 & ТР $53,64 \%$ & FLT3,67\% & & & \\
\hline & & & CNB & $40 \%$ & 10 uss & 32.1 & 40.9 & TP5314\% & FLT3,15\% & & & \\
\hline 19 & Lung & $\mathrm{ADC}$ & FNA $^{\mathrm{a}}$ & $30 \%$ & 2 smears & 0.4 & 4.3 & $E G F R, 27 \%$ & & & & \\
\hline & & & CNB & $25 \%$ & 8 uss & 14.6 & 27.3 & $E G F R, 14 \%$ & & & & \\
\hline 20 & $\mathrm{LN}$ & Lung ADC & FNA & $60 \%$ & 3 smears & 2.2 & 13.6 & NRAS,63\% & $S M O, 62 \%$ & ТР53,79\% & & \\
\hline & & & $\mathrm{CNB}$ & $40 \%$ & 13 uss & 42.4 & 40.9 & NRAS,53\% & $S M O, 56 \%$ & TP53, 67\% & & \\
\hline 21 & ST & UtCA & FNA & $25 \%$ & 3 smears & 3.2 & 13.6 & CTNNB1,7\% & $F B X W 7,7 \%$ & TP53,1\% & & \\
\hline & & & CNB & $30 \%$ & 8 uss & 24.8 & 27.3 & CTNNB1,17\% & FBXW7,17\% & TP53,9\% & & \\
\hline 22 & Liver & Pr ADC & FNA & $60 \%$ & 5 smears & 12.2 & 13.6 & ТР $53,60 \%$ & & & & \\
\hline & & & CNB & $22 \%$ & 13 uss & 1.5 & 13.6 & TP53,34\% & & & & \\
\hline 23 & $\mathrm{LN}$ & $\mathrm{Br} \mathrm{ADC}$ & FNA & $60 \%$ & 3 smears & 4 & 13.6 & PIK $3 C A, 48 \%$ & & & & \\
\hline & & & $\mathrm{CNB}$ & $25 \%$ & 10 uss & 51 & 54.5 & PIK $3 C A, 16 \%$ & & & & \\
\hline 24 & Liver & SqCC & FNA & $50 \%$ & 3 smears & 1.6 & 13.6 & TP53,33\% & & & & \\
\hline & & & CNB & $50 \%$ & 5 uss & 12.3 & 13.6 & TP53,69\% & & & & \\
\hline
\end{tabular}

Abbreviations: ADC adenocarcinoma; Adr, adrenal gland; Br CA, breast carcinoma; CNB, core needle biopsy; CRC, colorectal adenocarcinoma; Dx, diagnosis; FNA, fine needle aspiration; LN, lymph node; MAF, mutation allelic frequency; MM, malignant melanoma; PDC, poorly differentiated carcinoma; Pr ADC prostatic adenocarcinoma; Pt, Patient; RCC, renal cell carcinoma; SqCC, squamous cell carcinoma; ST, soft tissue; UroCA, urothelial carcinoma; uss, unstained slides; UtCA, uterine endometrial carcinoma.

Additional details of specific mutations detected in Supplementary Table 1.

${ }^{\mathrm{a}}$ Samples with $<10 \mathrm{ng}$ input DNA. 


\section{Discussion}

With advances in radiologic imaging techniques, the ability to detect and target smaller lesions using minimally invasive procedures has improved substantially. Although the volume of tissue acquired through these procedures is typically limited, the repertoire of molecular tests for predictive and prognostic markers necessary for clinical management is constantly increasing. ${ }^{18-23}$ Therefore, it is critical to judiciously utilize these limited tissue specimens for ancillary testing, including nextgeneration sequencing, to provide clinically relevant information.

Minimally invasive sampling methods including fine needle aspiration and core needle biopsy are frequently complementary and used concurrently as a diagnostic modality in a large number of institutions. Several studies have evaluated the feasibility of using cytologic fine needle aspiration samples for molecular testing and recent reviews have highlighted the advantages of cytologic specimens, especially non-formalin-fixed preparations, for molecular analysis. ${ }^{2,4,18,20}$ Aspiration cytology specimens can provide rapid on-site evaluation for adequacy assessments at the time of the procedure enabling acquisition of a better sample with appropriate triaging. ${ }^{20,24-29}$ Most cytologic specimen preparations, with the exception of cell blocks, are not formalin-fixed and therefore provide better quality nucleic acids without cross-linking artifact. ${ }^{30,31}$ In addition, aspirates of bone metastases are not subject to harsh decalcifying agents and provide a better source for nucleic-cid retrieval than decalcified histologic tissue. ${ }^{32}$ Despite these distinct advantages, historically cytologic specimens have been underutilized for molecular studies. Reasons that may explain the underutilization of fine needle aspiration specimens include: (1) reluctance of many laboratories to validate a wide variety of cytologic specimen preparations (eg, direct smears, cytospin preparations, liquid-based cytology) for molecular assays; ;,16,18 (2) reluctance of cytopathologists to sacrifice irretrievable archival cytologic material (eg, direct smears); ;,16 and (3) a general misconception among the clinical and pathology community regarding the potential for utilization of cytologic material for molecular testing. $4,18,33$

In this study, we reviewed 24 paired fine needle aspiration and core needle biopsy samples to compare next-generation sequencing in these limited volume samples. In addition, we morphologically evaluated 100 consecutive concurrently acquired fine needle aspiration and core needle biopsy malignant samples from image-guided procedures to compare overall cellularity and tumor fraction. Our results demonstrate that the overall cellularity of fine needle aspiration samples was significantly higher than the concurrent core needle biopsy samples, suggesting that aspiration cytology frequently provides higher cellularity samples than

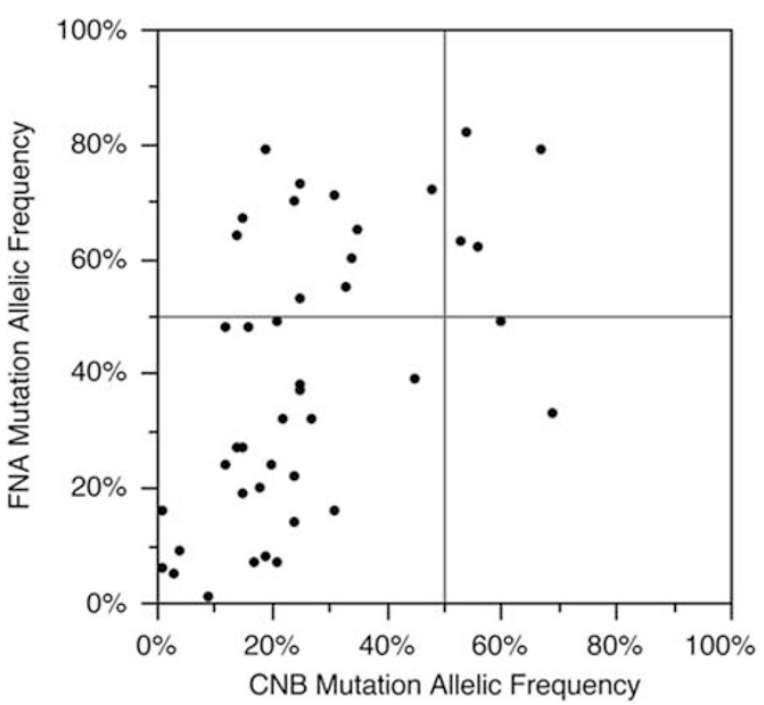

Figure 1 Comparison of concurrently acquired fine needle aspiration (FNA) and core needle biopsy (CNB) samples $(n=24)$, analyzed by next-generation sequencing (NGS).

needle biopsies for ancillary studies. Tumors with dyscohesive cells (eg, poorly differentiated carcinomas) and with extensive necrosis frequently yield high cellularity fine needle aspiration samples. Furthermore, our findings show that the tumor fraction in fine needle aspiration samples was significantly higher than that of the concurrent core needle biopsy samples. This finding reinforces the fact that specimens acquired via aspiration are highly enriched in tumor cells owing to the lower proportion of interfering stromal cells that are obtained in core needle biopsy samples. This is particularly true in tumors with increased amounts of sclerosis, eg, post-therapy cases with chemo/ radiation changes.

A higher fraction of core needle biopsy samples in this study, $\sim 14 \%$, failed to meet the minimum tumor fraction threshold of $20 \%$ for the next-generation sequencing assay. The concurrent fine needle aspiration sample in all these cases was deemed adequate (by cellularity and tumor fraction) for molecular testing. Review of the cases with subcentimeter nodules $(n=7)$ where the interventional radiologist may have performed the core needle biopsy prior to the fine needle aspiration, did not show any difference in cellularity or tumor fraction when compared to the cases with larger sized tumors (data not shown), indicating that the order of sampling may not necessarily affect the cellularity and/or tumor fraction of samples. Furthermore, the results of this study showed that cytologic smears from fine needle aspiration samples provided better sequencing metrics, despite lower DNA yields following tissue extraction. At our institution, the cytopathologist attempts to enrich for tumor content by delineating tumor-rich areas on the smears for manual microdissection. This approach is more 
Table 3 Next-generation sequencing metrics of paired fine needle aspiration and core needle biopsy samples

\begin{tabular}{|c|c|c|c|c|c|c|c|c|}
\hline \multirow[b]{3}{*}{ Patient } & \multicolumn{4}{|c|}{ Cytology FNA } & \multicolumn{4}{|c|}{ Surgical CNB } \\
\hline & \multirow[b]{2}{*}{ Failed amplicons } & \multirow[b]{2}{*}{ Total reads } & \multicolumn{2}{|c|}{ Average coverage } & \multirow[b]{2}{*}{ Failed amplicons } & \multirow[b]{2}{*}{ Total reads } & \multicolumn{2}{|c|}{ Average coverage } \\
\hline & & & All genes g $^{\mathrm{a}}$ & Selected genes ${ }^{\mathrm{b}}$ & & & All genes ge $^{\mathrm{a}}$ & Selected genes ${ }^{\mathrm{b}}$ \\
\hline 1 & 0 & 488774 & 1900 & 1896 & 1 & 449703 & 1812 & 1866 \\
\hline 2 & 0 & 430929 & 1812 & 2073 & 1 & 458819 & 1436 & 1681 \\
\hline 3 & 0 & 830160 & 3453 & 4462 & 0 & 539850 & 2365 & 2612 \\
\hline 4 & 0 & 515876 & 2277 & 2368 & 0 & 648144 & 2265 & 2361 \\
\hline 5 & 0 & 377856 & 1439 & 1523 & 0 & 557950 & 2266 & 2506 \\
\hline 6 & 0 & 531099 & 2326 & 2393 & 1 & 340523 & 1145 & 1196 \\
\hline 7 & 0 & 454462 & 1884 & 2076 & 1 & 613193 & 2568 & 2746 \\
\hline 8 & 2 & 436298 & 1612 & 1851 & 2 & 504483 & 1372 & 1466 \\
\hline 9 & 0 & 520394 & 2252 & 2582 & 0 & 331753 & 2602 & 2851 \\
\hline 10 & 1 & 528901 & 2358 & 2178 & 1 & 482935 & 2123 & 2124 \\
\hline 11 & 0 & 551501 & 2330 & 2634 & 2 & 483501 & 1524 & 1712 \\
\hline 12 & 0 & 446648 & 1940 & 2014 & 6 & 205109 & 860 & 919 \\
\hline 13 & 1 & 604682 & 2688 & 2541 & 2 & 289770 & 1221 & 1248 \\
\hline 14 & 0 & 617246 & 2786 & 2771 & 2 & 381829 & 1664 & 1675 \\
\hline 15 & 0 & 639483 & 2742 & 2967 & 1 & 329539 & 1409 & 1430 \\
\hline 16 & 0 & 470941 & 2097 & 2296 & 0 & 473185 & 2054 & 2328 \\
\hline 17 & 0 & 592983 & 2586 & 3443 & 0 & 427086 & 1851 & 2073 \\
\hline 18 & 2 & 450022 & 2022 & 2530 & 0 & 556847 & 2444 & 2694 \\
\hline 19 & 2 & 462336 & 2042 & 2172 & 1 & 419923 & 1833 & 1895 \\
\hline 20 & 3 & 420885 & 1878 & 2313 & 13 & 382637 & 1500 & 1782 \\
\hline 21 & 0 & 442214 & 1797 & 1937 & 18 & 350307 & 950 & 1102 \\
\hline 22 & 0 & 423767 & 1883 & 2281 & 0 & 413526 & 1775 & 1983 \\
\hline 23 & 0 & 388513 & 1741 & 2020 & 1 & 448429 & 1894 & 2066 \\
\hline 24 & 0 & 586757 & 2404 & 2646 & 1 & 447056 & 1896 & 2083 \\
\hline Mean & 0.46 & 509014 & 2177 & 2415 & 2.25 & 439004 & 1785 & 1933 \\
\hline s.d. & 0.88 & 100928 & 451 & 596 & 4.34 & 103827 & 494 & 541 \\
\hline
\end{tabular}

${ }^{\mathrm{a}}$ Normalized average amplicon coverage for all 50 genes analyzed was higher in FNA samples $P=0.025$. ${ }^{\mathrm{b}}$ Normalized average amplicon coverage for selected genes of relevance (BRAF, KRAS, NRAS, HRAS, EGFR, PIK3CA, KIT, PTEN, and TP53) was higher in FNA samples P=0.014.

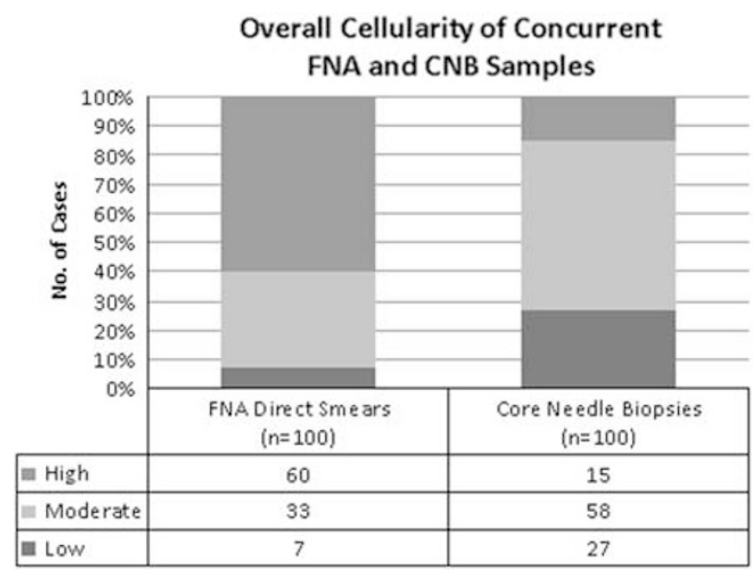

Figure 2 Comparison of overall cellularity (designated as high, moderate, and low) of fine needle aspiration (FNA) smears and concurrently acquired core needle biopsy (CNB) samples.

amenable to the three-dimensional clusters of cells on direct smears allowing for easier tumor mapping than the small cell block and core needle biopsy sections, which are usually microdissected in their entirety. This results in an increase of the tumor fraction, but at the cost of lowering the effective cellularity for tissue extraction. ${ }^{2}$ This may explain the significantly lower DNA yield $(P=0.01)$ and higher tumor fraction $(P=0.003)$ seen with the fine needle aspiration smears that were extracted and analyzed by next-generation sequencing. However, even with lower DNA yields, all fine needle aspiration samples had adequate DNA for successful nextgeneration sequencing analysis and were able to detect all mutations that were identified in the concurrent core needle biopsy sample.

Sample adequacy assessments for cellularity and tumor fraction are morphologic estimations performed by visual inspection under light microscopy and subject to inter-observer variability. ${ }^{34}$ Mutation allelic frequency by next-generation sequencing is an independent metric that can be used to quantify tumor fraction. Our results show that most fine needle aspiration samples had higher mutation allelic frequencies that corresponded with higher estimated tumor fractions seen in the samples. Fine needle aspiration samples also had lower numbers of failed amplicons and higher average amplicon coverage when compared with the concurrent core needle biopsy samples. These results indicate that cytology smears may provide better substrates for next-generation sequencing analysis than the concurrently acquired core needle biopsy specimens. Therefore, our results argue against reflexing all molecular test requests of image-guided procedures 
Table 4 Cellularity comparison of fine needle aspiration smears and core needle biopsies $(n=100)$ based on tumor type

\begin{tabular}{lcc}
\hline & FNA smears (\%) high cellularity & CNB (\%) high cellularity \\
\hline Adenocarcinoma $(n=51)$ & $25(49 \%)$ & $2(4 \%)$ \\
Squamous cell carcinoma $(n=18)$ & $14(78 \%)$ & $6(33 \%)$ \\
Poorly differentiated carcinoma $(n=11)$ & $9(82 \%)$ & $0(0 \%)$ \\
Melanoma $(n=7)$ & $2(29 \%)$ & $3(43 \%)$ \\
Neuroendocrine/small cell carcinoma $(n=8)$ & $8(100 \%)$ & $2(25 \%)$ \\
Others $^{a}(n=5)$ & $2(40 \%)$ & $2(40 \%)$ \\
\hline
\end{tabular}

${ }^{\text {a}}$ Others: sarcoma, germ cell tumor, and chordoma.

Table 5 Cellularity comparison of fine needle aspiration smears and core needle biopsies $(n=100)$ based on site of lesion

\begin{tabular}{llcl}
\hline Site of Lesion & Cellularity & $\begin{array}{c}\text { FNA smears } \\
(\%)\end{array}$ & CNB (\%) \\
\hline Lung $(n=34)$ & High cellularity & $19(56 \%)$ & $7(21 \%)$ \\
& Low cellularity & $3(9 \%)$ & $7(21 \%)$ \\
Liver $(n=23)$ & High cellularity & $17(74 \%)$ & $4(17 \%)$ \\
& Low cellularity & $1(4 \%)$ & $5(22 \%)$ \\
Bone/soft tissue & High cellularity & $8(35 \%)$ & $1(4 \%)$ \\
$(n=23)$ & & $3(13 \%)$ & $8(35 \%)$ \\
Lymph node & Low cellularity & $13(77 \%)$ & $2(12 \%)$ \\
$(n=17)$ & High cellularity & & \\
& Low cellularity & $0(0 \%)$ & $7(41 \%)$ \\
Others $^{\text {a }}(n=3)$ & High cellularity & $3(100 \%)$ & $1(33 \%)$ \\
& Low cellularity & $0(0 \%)$ & $0(0 \%)$ \\
\hline
\end{tabular}

${ }^{\mathrm{a} O t h e r s: ~ a d r e n a l ~ g l a n d ~ a n d ~ k i d n e y . ~}$

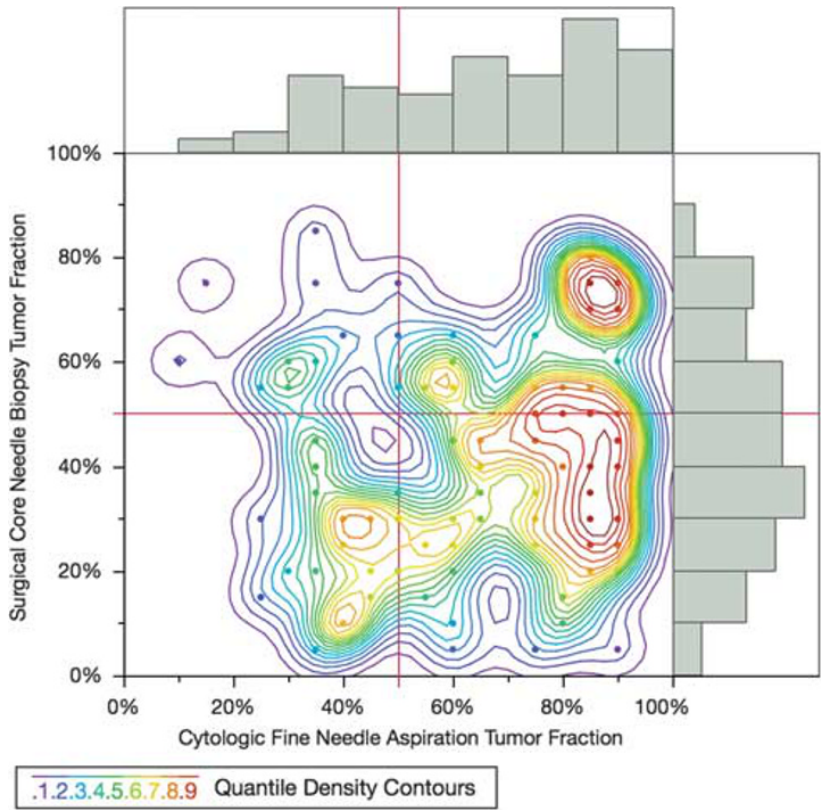

Figure 3 Density contour plot comparing estimated tumor fraction from concurrently acquired core needle biopsy and fine needle aspiration samples $(n=100)$.

to histologic biopsy specimens, especially in the context of a concurrently acquired aspiration cytology sample. Instead, molecular test requests on image-guided core needle biopsy samples need to be reviewed in conjunction with concurrently
Table 6 Morphologic estimation of tumor fraction in fine needle aspiration and concurrently acquired core needle biopsy samples

\begin{tabular}{lcc} 
Tumor fraction & FNA $(\mathrm{n}=100)$ & CNB $(\mathrm{n}=100)$ \\
\hline$<20 \%$ & 2 & 14 \\
$20-39 \%$ & 15 & 32 \\
$40-59 \%$ & 19 & 30 \\
$60-79 \%$ & 27 & 21 \\
$\geq 80 \%$ & 37 & 3 \\
\hline
\end{tabular}

Abbreviations: CNB, core needle biopsy; FNA, fine needle aspiration.

acquired fine needle aspiration specimens, if available, to select the best material for the downstream assay. The findings in this study are of considerable importance in the context of the 2013 College of American Pathologists/International Association for the Study of Lung Cancer/Association for Molecular Pathology (CAP/IASLC/AMP) clinical practice guidelines for molecular testing of lung cancer, that recommends the use of cell block sections over smears for all molecular testing. However, the guidelines are currently under revision and updated guidelines are expected to address the use of alternative cytologic preparations.

Molecular testing is now standard of care for the management of patients with several solid organ malignancies. Frequently, patients present with advanced stage disease that precludes excisional biopsy, and small specimens, including cytologic preparations, are often the only diagnostic material available for prognostic and predictive testing. Therefore, judicious use of all these limited volume specimens is needed to maximize utilization for clinically relevant biomarkers and minimize the need for re-biopsy. Many clinical trials requiring tissue for molecular studies prior to enrollment often do not accept cytology specimens as a tissue source, thus excluding patients from enrollment. ${ }^{33}$ The reasons for excluding cytologic tissue for molecular studies are manifold, some of which have been discussed above. Standardizing specimen handling and processing techniques across cytology laboratories is critical for easier validation of cytologic specimen preparations. Digital archiving by scanning or photographing archival slides can circumvent some of the issues associated with sacrificing 
cytologic smear slides for molecular testing. A better understanding and familiarity of the advantages and limitations of cytology specimens and the preanalytic factors that influence molecular testing is critical to influence this practice.

In summary, in this study we compared cytologic fine needle aspiration samples with concurrently acquired core needle biopsy samples. The results indicate that the cytology smears, as compared with core needle biopsy samples, have better cellularity, a higher tumor fraction, and better metrics by nextgeneration sequencing. The latter include higher mutation allelic frequency, higher average amplicon coverage, and lower numbers of under-performing amplicons. These results support the integration of cytologic specimens into routine molecular diagnostics workflow for maximizing limited tissue for clinically relevant genomic testing. The results presented also support incorporating cytologic specimens into clinical trials with the potential for increased patient enrollment and greater availability of tissues for subsequent molecular testing. In the era of precision medicine, cytopathologists can have a pivotal role in overseeing tissue sampling, processing, and appropriate triaging of small specimens to facilitate optimal utilization for molecular testing.

\section{Disclosure/conflict of interest}

The authors declare no conflict of interest.

\section{References}

1 Coley SM, Crapanzano JP, Saqi A. FNA, core biopsy, or both for the diagnosis of lung carcinoma: Obtaining sufficient tissue for a specific diagnosis and molecular testing. Cancer Cytopathol 2015;123:318-326.

2 Roy-Chowdhuri S, Stewart J. Preanalytic variables in cytology: lessons learned from next-generation sequencing. Arch Pathol Lab Med 2016;140:1191-1199.

3 Schneider F, Smith MA, Lane MC et al. Adequacy of core needle biopsy specimens and fine-needle aspirates for molecular testing of lung adenocarcinomas. Am J Clin Pathol 2015;143:193-200 quiz 306.

4 Bellevicine C, Malapelle U, de Luca C et al. EGFR analysis: current evidence and future directions. Diagn Cytopathol 2014;42:984-992.

5 Clark DP. Seize the opportunity: underutilization of fine-needle aspiration biopsy to inform targeted cancer therapy decisions. Cancer 2009;117:289-297.

6 Roy-Chowdhuri S, Goswami RS, Chen H, et al. Factors affecting the success of next-generation sequencing in cytology specimens. Cancer Cytopathol 2015;123: 659-668.

7 Smouse JH, Cibas ES, Janne PA et al. EGFR mutations are detected comparably in cytologic and surgical pathology specimens of nonsmall cell lung cancer. Cancer 2009;117:67-72.

8 Lozano MD, Zulueta JJ, Echeveste JI, et al. Assessment of epidermal growth factor receptor and K-ras mutation status in cytological stained smears of non-small cell lung cancer patients: correlation with clinical outcomes. Oncologist 2011;16:877-885.

9 Bozzetti C, Negri FV, Azzoni C, et al. Epidermal growth factor receptor and Kras gene expression: reliability of mutational analysis on cytological samples. Diagn Cytopathol 2013;41:595-598.

10 Sun PL, Jin Y, Kim H et al. High concordance of EGFR mutation status between histologic and corresponding cytologic specimens of lung adenocarcinomas. Cancer Cytopathol 2013;121:311-319.

11 Khode R, Larsen DA, Culbreath BC, et al. Comparative study of epidermal growth factor receptor mutation analysis on cytology smears and surgical pathology specimens from primary and metastatic lung carcinomas. Cancer Cytopathol 2013;121:361-369.

12 Bruno P, Mariotta S, Ricci A, et al. Reliability of direct sequencing of EGFR: comparison between cytological and histological samples from the same patient. Anticancer Res 2011;31:4207-4210.

13 Karnes HE, Duncavage EJ, Bernadt CT. Targeted nextgeneration sequencing using fine-needle aspirates from adenocarcinomas of the lung. Cancer Cytopathol 2014;122:104-113.

14 Rekhtman N, Brandt SM, Sigel CS, et al. Suitability of thoracic cytology for new therapeutic paradigms in non-small cell lung carcinoma: high accuracy of tumor subtyping and feasibility of EGFR and KRAS molecular testing. J Thorac Oncol 2011;6:451-458.

15 Billah S, Stewart J, Staerkel G et al. EGFR and KRAS mutations in lung carcinoma: molecular testing by using cytology specimens. Cancer Cytopathol 2011; 119:111-117.

16 Vigliar E, Malapelle U, de Luca C et al. Challenges and opportunities of next-generation sequencing: a cytopathologist's perspective. Cytopathology 2015;26: 271-283.

17 Kanagal-Shamanna R, Portier BP, Singh RR, et al. Nextgeneration sequencing-based multi-gene mutation profiling of solid tumors using fine needle aspiration samples: promises and challenges for routine clinical diagnostics. Mod Pathol 2014;27:314-327.

18 da Cunha Santos G, Saieg MA. Preanalytic parameters in epidermal growth factor receptor mutation testing for non-small cell lung carcinoma: a review of cytologic series. Cancer Cytopathol 2015;123:633-643.

19 Roh MH. The utilization of cytologic fine-needle aspirates of lung cancer for molecular diagnostic testing. J Pathol Transl Med 2015;49:300-309.

20 Knoepp SM, Roh MH. Ancillary techniques on direct-smear aspirate slides: a significant evolution for cytopathology techniques. Cancer Cytopathol 2013; 121:120-128.

21 Popper HH, Timar J, Ryska A et al. Minimal requirements for the molecular testing of lung cancer. Transl Lung Cancer Res 2014;3:301-304.

22 Thunnissen E, Kerr KM, Herth FJ, et al. The challenge of NSCLC diagnosis and predictive analysis on small samples. Practical approach of a working group. Lung Cancer 2012;76:1-18.

23 Aisner DL, Sams SB. The role of cytology specimens in molecular testing of solid tumors: techniques, limitations, and opportunities. Diagn Cytopathol 2012;40: 511-524.

24 Fassina A, Corradin M, Zardo D et al. Role and accuracy of rapid on-site evaluation of CT-guided fine needle aspiration cytology of lung nodules. Cytopathology 2011;22:306-312. 
25 Baram D, Garcia RB, Richman PS. Impact of rapid onsite cytologic evaluation during transbronchial needle aspiration. Chest 2005;128:869-875.

26 Collins BT, Chen AC, Wang JF et al. Improved laboratory resource utilization and patient care with the use of rapid on-site evaluation for endobronchial ultrasound fine-needle aspiration biopsy. Cancer Cytopathol 2013;121:544-551.

27 Collins BT, Murad FM, Wang JF et al. Rapid on-site evaluation for endoscopic ultrasound-guided fine-needle biopsy of the pancreas decreases the incidence of repeat biopsy procedures. Cancer Cytopathol 2013;121:518-524.

28 da Cunha Santos G, Ko HM, Saieg MA et al. "The petals and thorns" of ROSE (rapid on-site evaluation). Cancer Cytopathol 2013;121:4-8.

29 Nakajima T, Yasufuku K, Saegusa F, et al. Rapid on-site cytologic evaluation during endobronchial ultrasoundguided transbronchial needle aspiration for nodal staging in patients with lung cancer. Ann Thorac Surg 2013;95:1695-1699.
30 Williams C, Ponten F, Moberg C, et al. A high frequency of sequence alterations is due to formalin fixation of archival specimens. Am J Pathol 1999;155: 1467-1471.

31 Srinivasan M, Sedmak D, Jewell S. Effect of fixatives and tissue processing on the content and integrity of nucleic acids. Am J Pathol 2002;161:1961-1971.

32 Singh VM, Salunga RC, Huang VJ, et al. Analysis of the effect of various decalcification agents on the quantity and quality of nucleic acid (DNA and RNA) recovered from bone biopsies. Ann Diagn Pathol 2013;17:322-326.

33 Roy-Chowdhuri S, Aisner DL, Allen TC, et al. Biomarker testing in lung carcinoma cytology specimens: a perspective from members of the pulmonary pathology society. Arch Pathol Lab Med 2016;140:1267-1272.

34 Viray H, Li K, Long TA, et al. A prospective, multi-institutional diagnostic trial to determine pathologist accuracy in estimation of percentage of malignant cells. Arch Pathol Lab Med 2013;137:1545-1549.

Supplementary Information accompanies the paper on Modern Pathology website (http://www.nature.com/ modpathol) 